\title{
Design and Implementation of GeoScience Case Database on JSF Frame for Teaching
}

\author{
Zhenhe Zhao ${ }^{1}$, Shilai Wang ${ }^{1}$, Ping Zhang ${ }^{\star^{1, a}}$ \\ ${ }^{1}$ Geo-Exploration Science and Technology College, Jilin University, Changchun 130061, China \\ * Corresponding author: Ping Zhang \\ ${ }^{a} z p @ j l u . e d u . c n$
}

Keywords: JSF framework; GeoScience Case Database; Responsive Layout; BootsFaces; PrimeFaces

\begin{abstract}
Case base construction is of great significance for the promotion of case teaching. But at present there is no successful geosciences case library can be used as reference. So the construction and development of geosciences case library has significance to fill the gap. This article is about design and implementation of geosciences case database based on the JSF framework, PrimeFaces component and BootsFaces component library. This case bank not only has some functions, such as case search, case browse, case upload, case download, user interaction, but also can set videos, audios, pictures and texts in one. In particular, this geoscience case database can be running on both of PC side and mobile terminal. Therefore the geoscience case database has good prospects in application and promotion.
\end{abstract}

\section{Introduction}

Case teaching originated in 1870 in the United States at Harvard Law School [1], which is education according to the education goal, to case as the basic teaching material, learners will be introduced in the situation of educational practice, through the forms of teachers and students between the multidirectional interaction, improve learners face complicated educational context of the ability to make decisions and actions [2]. In 1921, Harvard Business School implemented the case teaching formally and established the specialized agencies to do the case development and case studies [1]. After years of improvement and promotion, Harvard Business School's case library has a great impact in the world, the case teaching relying on the case library has been competing around the world to follow $[3,4,5]$. This trend has been expanded from the field of law, medicine, business administration and other more disciplines. Case is the core content and the fundamental guarantee of case teaching, so case base construction is of great significance for the promotion of case teaching [6].

Case studies in the field of education in China are not many, and individual university's case library is still in the early stages of construction and trial operation. Dalian University of Technology established the first case library in China in 1987 and founded the China Case Sharing Center in 2007 [6]. This is the largest business management teaching case library of China. Tsinghua University set up the China Business Management Case Center in 1999. Peking University founded Peking University Management Case Research Center in 2000 [6]. But in the field of geoscience both at home and abroad, case study and development is rare; case development standards have not yet formed; the number of high-quality case are scarce. There is no success of geosciences case library can be used as reference. So the construction and development of geosciences case library has significance to fill the gap.

Moreover, compared to the latest development in case library abroad, there are several problems in case library of China as follows. ( i ) Case library page layout is not responsive layout, which can only be displayed on the PC side normally, but cannot adapt to the normal display of different size screens. This would bring inconvenience to the case study for the learners with the mobile terminal. (ii) Case library does not provide an interactive module for learners. Thus the distance learners cannot put forward and solve their problems in the study on the Internet. This would be not conductive to the second excavation and utilization of case library. (iii) Case display forms is single, most of which is in 
text and a small number of pictures. This would affect the learning interest and learning effect for learners.

It is gratifying that the continuous development of network technology, programming is becoming more and more complex, which brings great challenges to the developers, but also brings opportunities to the development of the ideal case library. For example, JSF is a better framework to realize a network case database, which is a Java web framework based on the user page. JSF not only provides a component-development model, but also reduces the developer's burden at the end of the web effectively. This would enable developers to concentrate on the development of business logic layer, thus improving the efficiency of development [7]. Based on Bootstrap framework component library, BootsFaces in JSF framework can achieve responsive layout at the end of web and can be well combined with JSF [8, 9]. This article is about the establishment of teaching case database on geoscience exploration and information technology bases on the JSF framework, which can be running on both of the PC side and the mobile terminal. This case database can realize some functions, such as case upload, case download, case browse, case search, user interaction.

\section{Case Database Analysis and Design}

User Requirement Analysis. The construction of this geoscience case base is the spirit of the document of the Ministry of education on improving and strengthening the construction of postgraduate courses (No. [2014] 5). This case base is oriented to the geo-exploration and information technology major and other related postgraduates in Jilin University, whose goal is to improve the quality of postgraduate education, to enhance the effectiveness of case teaching, and to promote the development of continuous education informatization. The contents of the case base consists of three second level disciplines: geo-exploration and information technology, cartography and geographic information system (GIS), cartography and geographic information engineering. The case library can support several case formats, for example, video, audio, text, pictures etc. The main functions of the case base include case upload, case download, case search, case browse, and user interaction (Fig. 1).

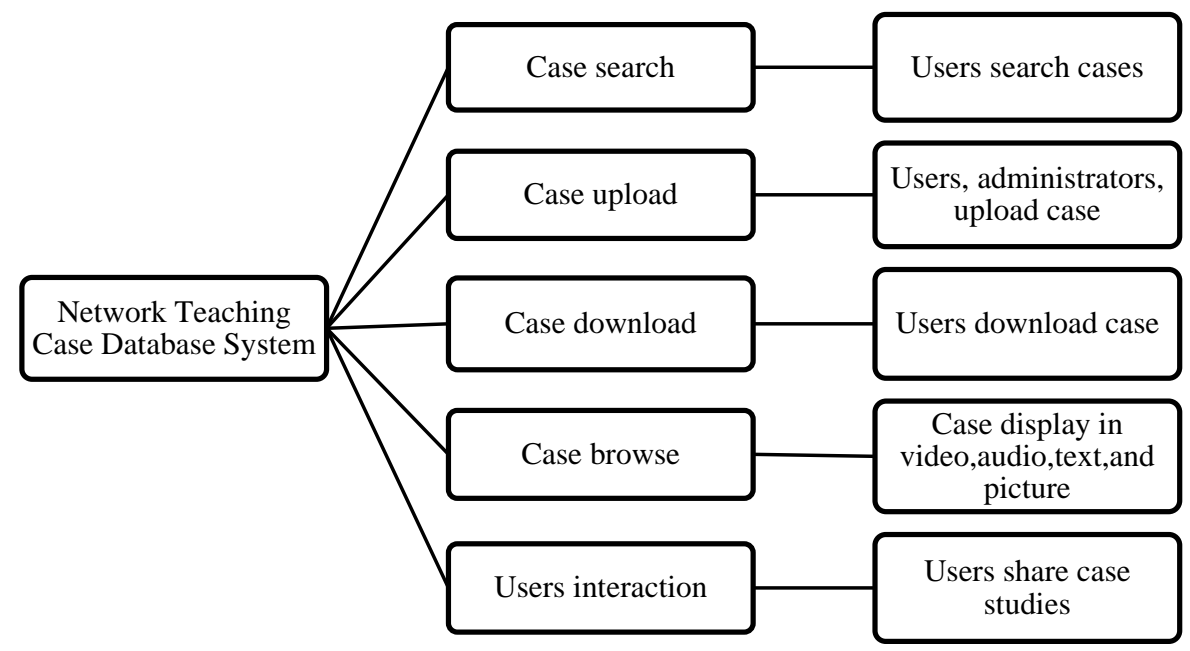

Fig. 1 Function design of the geoscience case database.

Function Analysis. User management is the most basic function for network case database, which should easily manage user registration and user login. There are three types of users for the case base here, including postgraduate students, teachers and administrators. Postgraduate students are the main user groups. As long as the user registration has been passed by the administrator, user can get access to the case base. After landing from the teacher's login screen, teachers can be involved in managing case resources, such as case review, case upload and case delete. Teachers can also 
participate in interactive communication with students. After landing on the administrator interface, administrator can do the authentication of user registration information and update the user information.

The case is the case resource management can be an important requirement for the survival and development of a case library, because it can implement some important functions, such as case search, case upload, case review, case download, case delete, and case collect etc. General users (postgraduate students and teachers) can search, upload, download, review, and collect case. Administrators can audit the case that the ordinary users have uploaded, upload or delete cases by themselves directly.

Case evaluation is an important part in the case teaching activities since effective case evaluation can stimulate the growth and progress of learners. Case evaluation is mainly about the effectiveness evaluation of the learner to the case, which can be used as a new kind of resources by other learners.

User interaction is also an essential feature of the case library because case library does need to publish the latest announcement for each learner. Administrators can add or delete the outdated announcements according to the actual needs; learners could display the corresponding announcements. In addition, general users can realize the communication among users in the exchange module, which can not only put forward their own problems for a case, but also can share the harvest of their own learning case.

Feasibility Analysis. JavaServer Faces (JSF) is a new standard Java framework for building Web applications. It provides a component-centric method for developing Web Java server end user interface, which simplifies the development process [10]. Meanwhile JSF is also strictly following the framework of model-view-controller (MVC) design pattern. It can clearly separate the client code (View) and application data industry logic code (model), which makes the JSF application has a higher maintainability and is more convenient to manage [11].

BootsFaces was the project that Riccardo Massera initiated and ended with in 2013, which was a new JSF component library based on the Bootstrap and jQuery UI enterprise application development to simplify the front end [12]. BootsFaces have the same grid system as Bootstrap, with a responsive layout feature. The responsive features of BootsFaces could help developers design only one version of case library website, which could adapt to mobile phones, small or big tablets, and desktop screens. Moreover, BootsFaces also supports sixteen kinds of Bootswatch themes, and can be integrated with PrimeFaces, OmniFaces, JSF and other ButterFaces components library [13].

PrimeFaces is an open source JSF component library, which has a wealth of functional components. It can not only meet the function requirements of the system of network teaching case library, but also can provide the detailed development documents and examples for developers [14]. It has more than thirty kinds of UI theme for developers to use. Thereby achieving network teaching case database is technically feasible. Thus it is feasible to realize the network case base at the technical level.

System Design. Currently there are many applications in java web framework. By comparison, we found that the JSF framework is in the true sense of the MVC pattern, with the development of high efficiency and the characteristics of heavy light client server. The support of a large number of open source component library makes JSF even stronger and at the same time JSF meets JavaEE stardards. Primefaces is committed to the creation of the final JSF component library, which is currently the most popular use of the JSF component library. BootsFaces is a relatively young JSF component library, with the grid system and Bootstrap, which can achieve rapid response type layout to have a good view effect. Selecting the JSF framework and then combining with two lightweight component libraries-Primefaces and Bootsfaces [15], which can realize the complex web applications and have good view effect at the client terminal. This can also adapt to different size of the screen response layout. MVC pattern of the system was designed as follows (Fig. 2). 


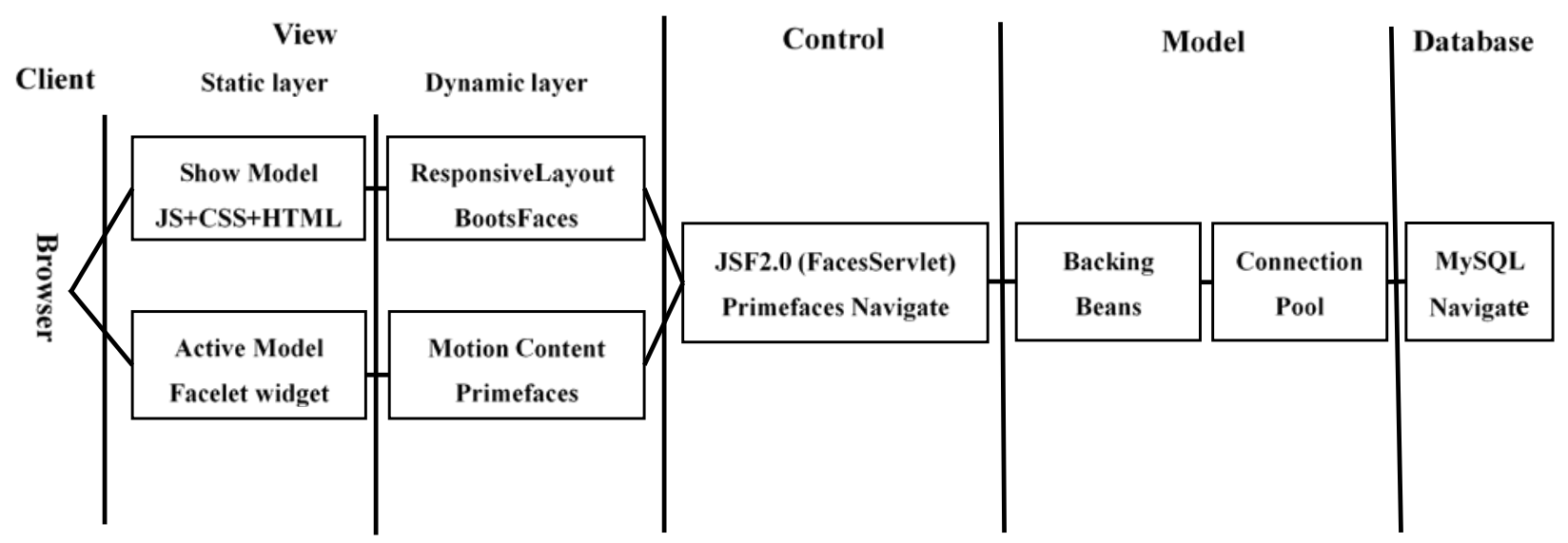

Fig. 2 System structure chart of the geoscience case database.

View. The presentation layer is responsible for the client trigger event to send the HTTP request and display the returned result view to the user $[16,17]$. This presentation layer design begins with the page layout and the function module. In order to achieve normal display of the Web page in different client terminal, as well as to show a better effect of the page, JSF component library Bootsfaces was used to design the page layout. Bootsfaces' grid system can realize the responsive layout. Relative to the layout of the Bootstrap, Bootsfaces code is more concise, and has a high efficiency of the layout. Primefaces component library is relatively more mature in the function module, which has been updated from the original version 2.2 to 5.3, and the function is more and more richly. It can meet the demands of the case base system here, whose fileupload, filedownload, media and other plug-ins have played an important role here.

Controller. The control layer is mainly responsible for intercepting the requests of the client, then passing the request to the business logic layer, and returning the results of the business logic layer to the client display $[16,17]$. The control layer of the case base here is mainly composed of JSF 2.0 FacesServlet and Primefaces navigate.When the client triggering the event, FacesServlet intercepts the user's HTTP requests to verify the request and use the beans backing call in the model to returen to the client display [15]. FacesServlet manages the JSF life cycle of JSF, configures FacesServlet in the $<$ servlet $></$ servlet $>$ tag of Web.xml configuration file, configures the Primefaces component navigation in the $<$ filter $></$ filter $>$ tag.

Model. The model layer is used to operate and implement the business logic layer of JSF, mainly through Java beans to process messages from the view layer and stored in the database. The model can be reused and composed primarily of backing beans and data persistence layer [16, 17]. This case base consists of multiple backing beans and database connection pool, connecting the front component trigger event behaviour with the back end beans backing by expression of \#\{\} [15]. Backing beans do the operations of data insert, search and save to database through the database connection pool, and then through the controller layer will be the corresponding results in the form of XHTML and form a view to return to the view layer, and finally in the client display.

\section{Case Database Implementation}

This case database was developed in the NetBeans8.0 environment, using component libraries of PrimeFaces 5.3 theme library, Bootstrap 1.01, PrimeFaces 5.3 and BootsFaces 0.8.1 version.

Implement of the Presentation Layer. The case library here used JSF2.0 Facelet page, which is the standard XHTML Web page. First loading the jar in the NetBeans8.0 environment component library file, then in the Web.xml <context-param $><$ /context-param $>$ tag configuring component library theme. Facelet page structure was set as follows.

$<$ html xmlns="http://www.w3.org/1999/xhtml"

xmlns:h="http://java.sun.com/jsf/html"xmlns:ui="http://java.sun.com/jsf/facelets" 


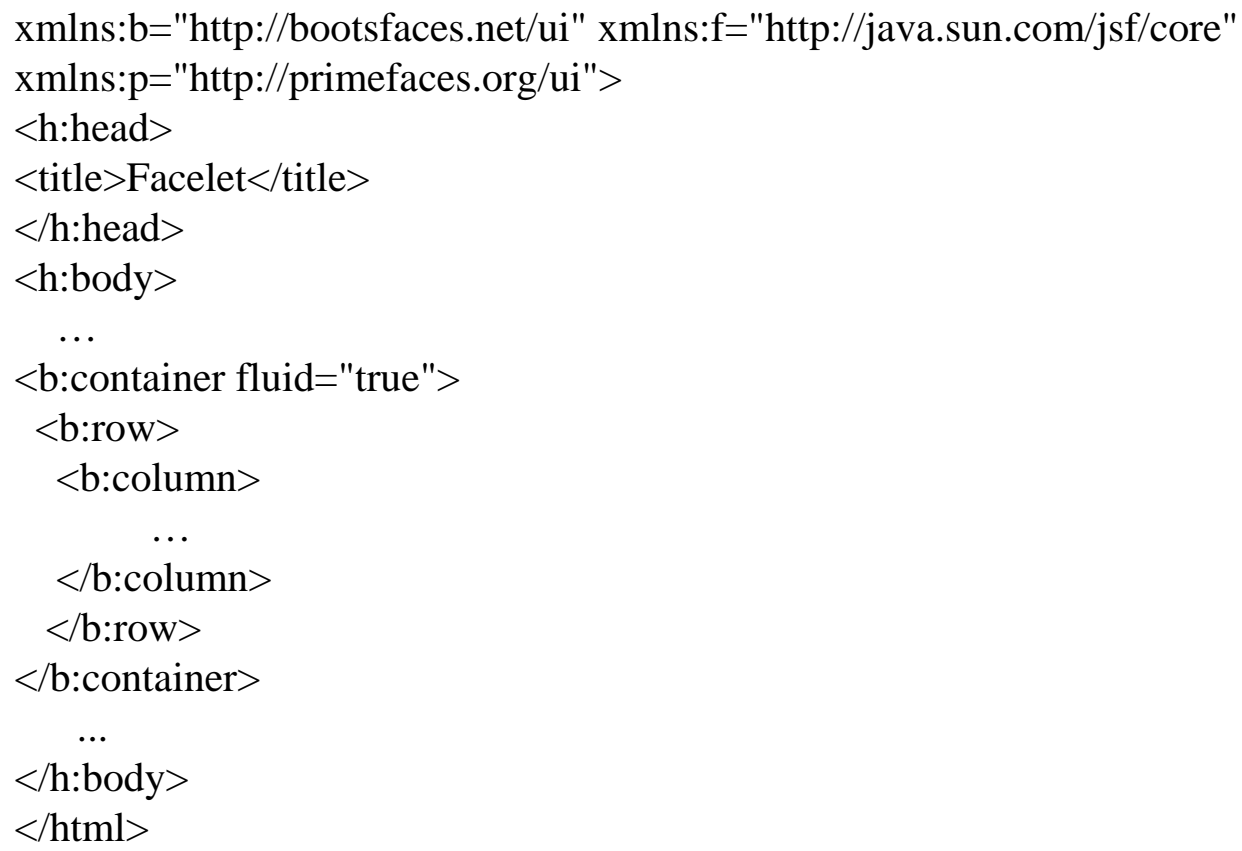

First Facelet page $<$ html $>$ tag introduced in JSF tags and BootsFaces tags and PrimeFaces tags, then $<$ h: body $></ \mathrm{h}$ : body $>$ tags used BootsFaces layout, the main applying the BootsFaces grid system, including $<$ b: container $>$, $<$ b: row $>$, $<$ b: column $>$ three tags. $<$ b: container fluid $=$ "true" $>$ tag of BootsFaces was equivalent to the Bootstrap $<$ div class $=$ "container-fluid" $>$ represents the container of grid system; <b: row $>$ tag contained in $<$ b: container $>$ tag represents the line in a grid layout, which represents the number of pages will be split into several lines; <b: column> tag contained in $<\mathrm{b}$ : row $>$ tag represents the column in a grid layout, which represents the number of line will be split into several columns. In the $<$ b: column $>$ tag to embed PrimeFaces tag $<\mathrm{p}$ : $>$, it realizes the main functions.

Implementation of Control Layer. In the JSF framework FacesServlet responsible for front-end controller, all user requests to go through FacesServlet before moving on to the next stage, through web.xml file for FacesServlet be configured as follows:

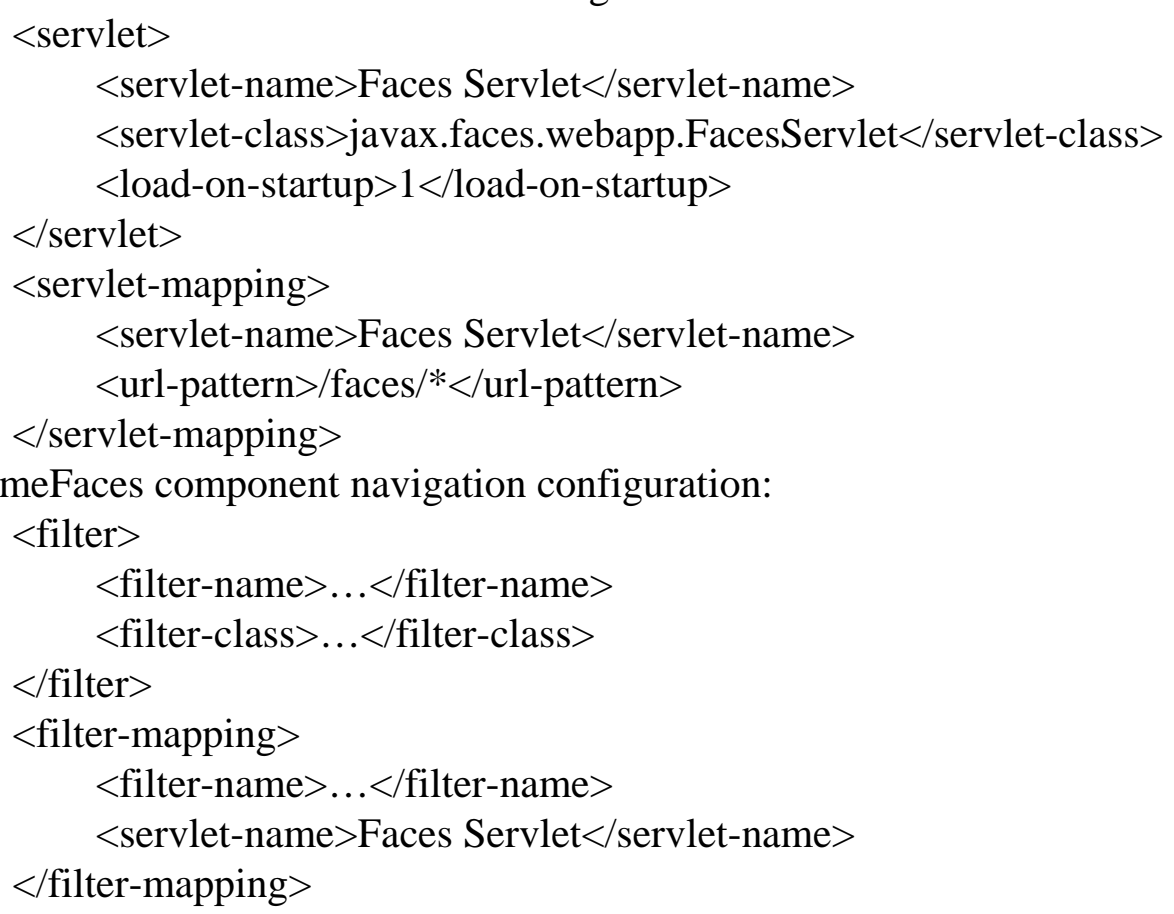

Implementation of Model Layer. Model layer composed mainly of backing beans to achieve the main function of the library network case database. Use primefaces plug in the presentation layer example fileupload plug, in Facelet page insert <p: fileUpload fileUploadListener = "\# 
\{Upload.handleAnliFileUpload $\} "$ mode = "advanced" dragDropSupport = "false" multiple = "true" sizeLimit = "10000000" fileLimit = "6" /> tag. By the Expression Language fileUploadListener = "\# \{Upload.handle Anli FileUpload\}" implementation calls for the model layer handleAnliFileUpload Java bean. sizeLimit attribute represents byte limit upload file, fileLimit attribute indicates the restrictions on the number of one-time upload files. Model Layer: Create handleAnliFileUpload Java bean class:

public void handleAnliFileUpload() \{

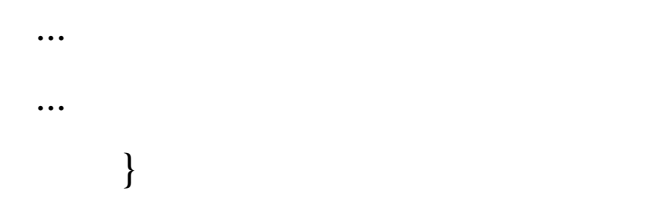

\section{Show Page.}

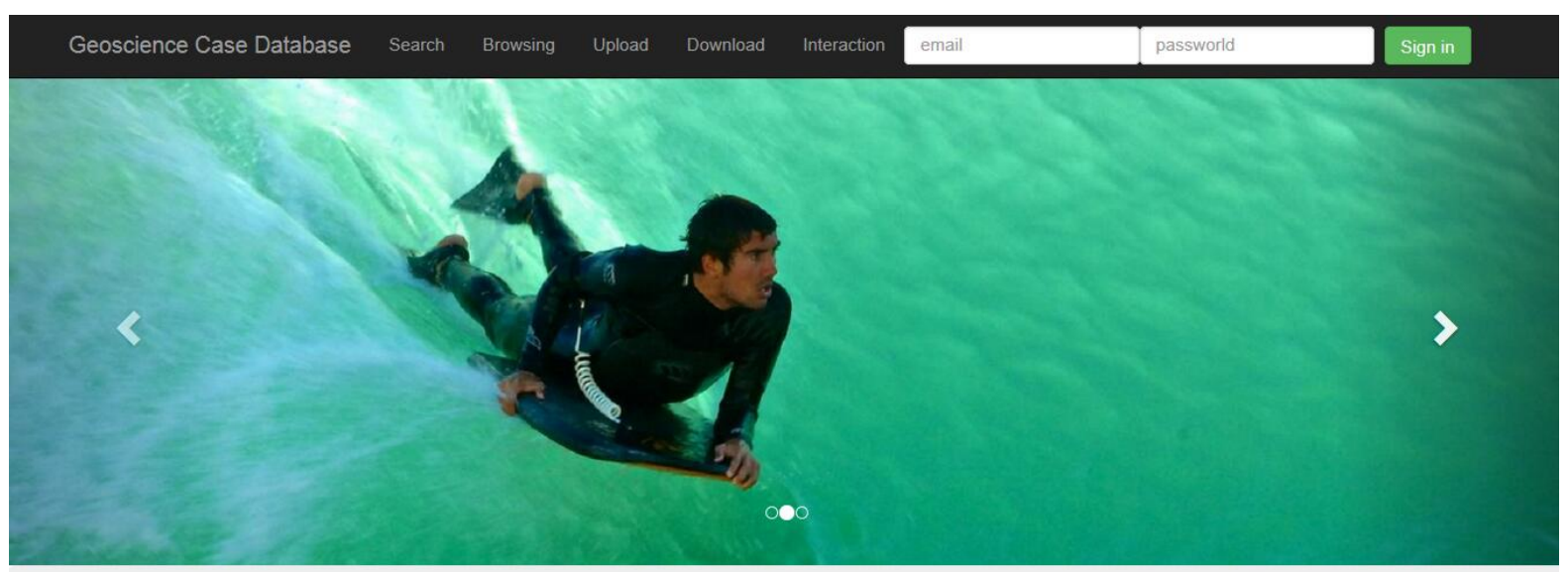

Welcome!

Fig. 3 Home page of the geoscience case database on PC side.
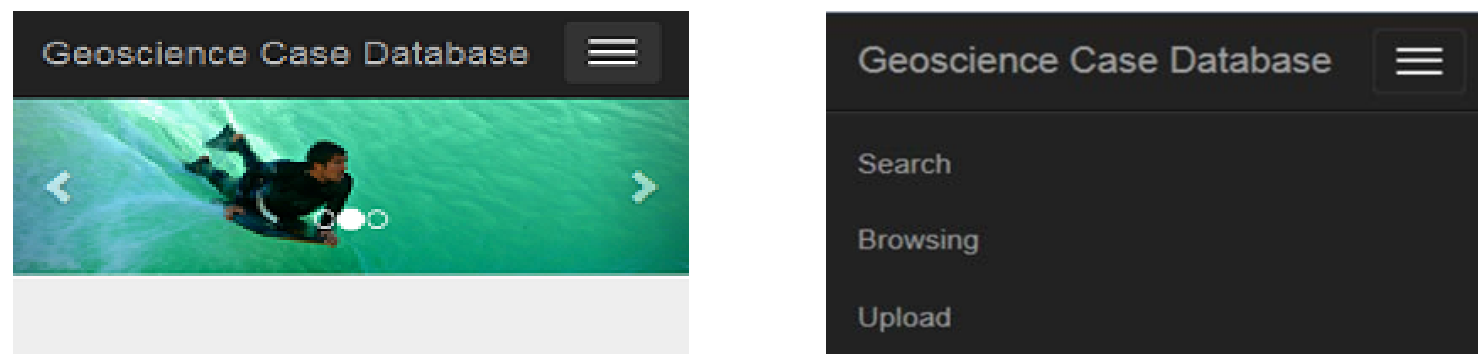

Welcome!

Download

Geoscience Case

Database can set videos, audios, pictures and texts

in one. In particular, this geoscience case database can be running on both of $\mathrm{PC}$ side and mobile terminal

\section{Learn more}

Interaction

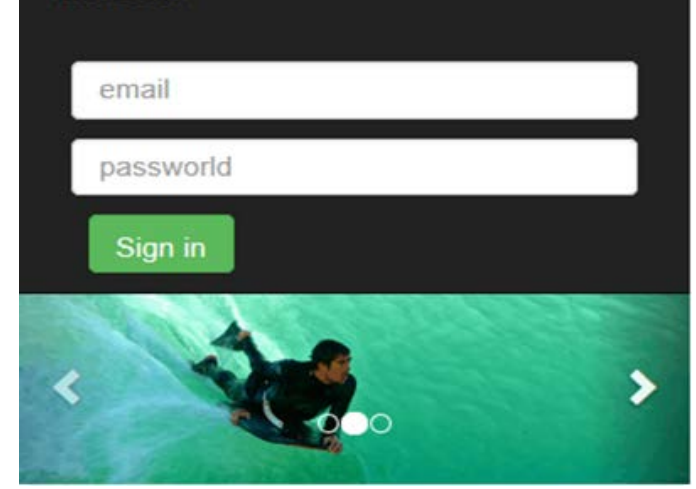

Fig. 4 Home page (left) and the navigation items (right) of the geoscience case library on mobile terminal. 


\section{Conclusion}

This study was about the design and implementation of the geoscience case database of Jilin University based on the MVC pattern and component library combination of Primefaces and Bootsfaces in JSF framework. This case library can set video, audio, text and picture in one, which makes learns receive information in the visual and auditory. This could not only enhance the effect of case teaching, but also improve the learning interests of learners. The case library can be achieved on both of the PC side and the mobile terminal, that is, learners can not only browse the case library on the PC side, but also through the mobile terminal if their registration information has been passed. This style can make good use of time for learners. The case library also can realize user interactions, which users can send their posts, communicate and solve learning problems at the interactive communication platform. Thus the case library has good prospects in application and promotion.

However, the geoscience case database has some disadvantages as well. Due to the JSF framework to the server requirements are relatively high, so the geoscience case database only in relatively high configuration servers, can play high running efficiency. In addition, the geoscience case database currently only supports Google and Firefox browsers, low version of the IE browser is still debugging. Not only that, JSF multimedia control stream file in binary analysis cannot be properly and only Java background transferring binary stream to use relative path references can work wellunder the project. The life cycle of JSF pages causes uncontroble multi cache influence for users controlling pages. We will continue to develop and improve the case library in the fure work in order to make the geoscience case database become an excellent example in the field of geoscience.

\section{Acknowledgements}

We are grateful for Jilin University to support this research by Postgraduate Education Reform Funding (No. 4500914A3103; 450060211402).

\section{References}

[1] G. F. Yang, H. J. Zhang: Foreign Elementary and Middle School Education. Vol. 6 (2008), p. 1-5. (In Chinese)

[2] J. Y. Sun: Case Teaching (Tianjin Education Press, China 2004). (In Chinese)

[3] D. Y. Lina: Journal of the Scholarship of Teaching and Learning. Vol. 13 (2013), p. 101-115.

[4] Y. Song: Research on the construction of the case base of public administration in China (Postgraduate Degree Paper of Xiangtan University, China 2010). (In Chinese)

[5] M. Li: Case teaching study of Harvard University (Postgraduate Degree Paper of Shenyang Normal University, China 2012). (In Chinese)

[6] Q, Yang: Research on case teaching and case base construction in international Chinese teacher education (Postgraduate Degree Paper of Minzu University of China 2012). (In Chinese)

[7] F. Wang, Q. R. Jiang, H. J. Yu: Computer Engineering and Design. Vol. 11(2007), p. 5221-5224.

[8] W. William: Electronic Design. Vol. 7 (2004), p. 55.

[9] M. H. Baturay, M. Birtane: Procedia - Social and Behavioral Sciences. Vol. 106(2013), p. 2275-2279.

[10] N. Jain: Journal of Global Research in Computer Science. Vol. 5(2014), p. 6-7.

[11] Information on http://www.bootsfaces.net 
[12] X. J. Wu, L. J. Ling: 2010 Second International Workshop on Education Technology and Computer Science. (2010), p. 766-768.

[13] Information on http://www.primefaces.org

[14] B. Dudney, J. Lehr, B. Willis, L. Mattingly: Mastering JavaServer Faces.(Wiley Publishing Inc, USA 2004).

[15] A. Goncalves: Beginning Java EE 7. (Apress, USA 2013).

[16] S. Li, L. Sun: 2011 International Conference on Computer Science and Service System. (2011), p. 2008-2010.

[17] X. Zhang, G. Cheng: 2008 International Conference on Computer Science and Software Engineering. (2008), p. 148-150. 